\title{
Neuromodulation Treatments for Schizophrenia
}

\section{Mehmet E. Dokucu, MD, PhD}

\author{
Address \\ Department of Psychiatry and Behavioral Sciences, Northwestern University \\ Feinberg School of Medicine, 710 North Lake Shore Drive \#1324, Chicago, IL \\ 60611, USA \\ Email: m-dokucu@northwestern.edu
}

Published online: 3 July 2015

(C) Springer International Publishing AG 2015

This article is part of the Topical Collection on Schizophrenia and Other Psychotic Disorders

Keywords Schizophrenia - Neuromodulation - Electroconvulsive therapy - Transcranial magnetic stimulation - ECT · TMS

\section{Opinion statement}

Schizophrenia is a psychiatric disorder that causes great suffering and debilitation worldwide. We have a multitude of medications that are effective for psychosis. However, these have not been successful in treating the negative and cognitive symptom domains of schizophrenia. These symptoms are responsible for the larger part of functional impairments that result from schizophrenia. In addition, there are many patients for whom no significant improvement is achieved even in the positive symptom domain. Hence, other treatment modalities have been explored to help these patients. Electroconvulsive therapy and transcranial magnetic stimulation are two of the most promising adjunct treatment methods for medication-resistant schizophrenia. Electroconvulsive therapy is the gold standard treatment for catatonias whether associated with schizophrenia, mood disorders, or other non-psychiatric disorders. Although not effective for negative symptoms, electroconvulsive therapy provides substantial augmentation to antipsychotic medications in improving positive symptoms and overall severity. Electroconvulsive therapy should be considered more often in patients with inadequate response to antipsychotic medications even when they do not have prominent affective symptoms. Transcranial magnetic stimulation has emerged as a promising useful therapeutic tool in targeting medicationresistant auditory hallucinations and negative symptoms. Transcranial magnetic stimulation has proven to be very safe and well-tolerated by the patients in spite of its labor intensiveness. The incorporation of transcranial magnetic stimulation to routine clinical use awaits further studies to substantiate its efficacy and to optimize and customize treatment parameters to individual patients and their symptom patterns. Moreover, combining transcranial magnetic stimulation with electroconvulsive therapy to synergize their likely different mechanisms of action is another exciting possibility. 


\section{Introduction and background}

Schizophrenia is a psychiatric disorder affecting and impairing approximately $1 \%$ of the population worldwide. It is a heterogeneous illness with multiple symptom dimensions: positive (hallucinations and delusions), negative (blunted affect, anhedonia, social deficits etc.), cognitive (working memory, executive functioning etc.), and movement (catatonia) domains. Antipsychotic medications are the treatment of choice for schizophrenia, but they improve the symptoms of the positive psychotic domain most effectively whereas cognitive and negative symptom domains have been more persistently resistant to treatments. As cognitive and negative symptom domains are strongly linked to poor functional outcomes in schizophrenia, finding and evaluating alternative interventions is a crucial aim of the ongoing research efforts. These included electroconvulsive therapy (ECT) (which happened to be a first-line treatment before the emergence of antipsychotic medications) and psychotherapies (individual and group) with limited success. There are now emerging novel device-based neuromodulation therapies that promise to overcome the shortcomings of the available treatment modalities. These include transcranial magnetic stimulation (TMS), transcranial direct current stimulation (tDCS), deep brain stimulation (DBS), vagus nerve stimulation (VNS), magnetic seizure therapy (MST), low field magnetic stimulation (LFMS), and many others. In addition to ECT, two of them (TMS and tDCS) have attracted the attention of researchers and distinguished themselves by the amount of research efforts. ECT and TMS have the largest amount of research conducted, and TMS is the next therapeutic modality that is closest to routine clinical use for schizophrenia patients.
In this article, we will review the selected clinically relevant literature evaluating the utility of non-invasive therapeutic neuromodulation modalities of electroconvulsive therapy and transcranial magnetic stimulation. Since these treatment modalities may differentially affect symptom domains (not unlike medications), when relevant for the treatment modality, we will review them within the context of the symptom domains, e.g., auditory hallucinations, catatonia. Currently, there are no studies reporting the combined use of therapeutic neuromodulation modalities for schizophrenia in controlled studies. Since these therapies have been more commonly used in major depressions, a few controlled studies of combined use exist for these disorders.

Up to a quarter of patients do not respond adequately to standard antipsychotic drug therapy [1]. According to the Clinical Antipsychotic Trials of Intervention Effectiveness (CATIE) study, up to $75 \%$ of patients had to change to another antipsychotic medication due to nonresponse or poor tolerability [2]. Clozapine is the preferred medication for treatment-resistant schizophrenia. However, between 25 and $50 \%$ of treatment-resistant or intolerant patients do not respond to Clozapine eithereven after 1 year of treatment [3]. For these patients, for those who are unable to use Clozapine and for patients whose clinical condition is too severe or acute for antipsychotic medications only, ECT has been reported to be a beneficial adjunct/augmentative treatment $[4 \bullet \bullet]$. However, negative public perception, cognitive adverse effects, and lack of availability limit the utility of ECT. Hence, other therapeutic brain stimulation modalities have been studied for treatmentresistant schizophrenia.

\section{Electroconvulsive therapy}

In the USA, the main indication for ECT is depression. However, ECT is utilized more widely (up to $88 \%$ ) for schizophrenia and psychotic disorders than for depression in Eastern Europe and most of Asia and Africa [5, 6]. Moreover, in many countries, ECT is used as a first-line treatment for all indications. This is likely due to a combination of lesser access to antipsychotic medications, other budgetary constraints, and diagnostic bias (i.e., lower rate of depression diagnosis) [5]. It is unclear how the stigma associated with ECT changes from one country to another and how this effects the variation of its utilization in different health care systems. 
Catatonia is a behavioral disorder of movement abnormalities that are characterized by numerous heterogeneous psychomotor signs. The most common signs are stupor, immobility, rigidity, and mutism. It can be caused by schizophrenia or the pharmacological treatment of schizophrenia (antipsychoticinduced catatonia can be overlapping with and/or indistinguishable from neuroleptic malignant syndrome) in addition to other neuropsychiatric and other medical disorders [7]. ECT and benzodiazepines are effectively used as first-line treatments regardless of what the presumed underlying etiology is [8]. There are no randomized clinical trials for the treatment of catatonias although some authors have suggested that catatonia associated with an affective disorder may have higher ECT response rates than with schizophrenia [9] although this is disputed by others [10]. The current treatment approaches are based on openlabel studies, retrospective analyses, and case reports of up to $100 \%$ response rates (e.g., see [11]). Moreover, catatonia is the only domain in schizophrenia where ECT is commonly utilized as monotherapy.

In acute catatonia, the ECT duration required for response is $2-5$ days in contrast to ECT for the treatment of mood disorders or schizophrenia in the absence of catatonia [7]. There are no well-established predictive criteria for ECT response to catatonia in schizophrenia patients. There is also less enthusiasm for the efficacy of ECT in schizophrenia with chronic catatonic behavior [12].

\section{Positive symptoms}

According to a comprehensive meta-analysis by Tharyan and Adams for the Cochrane Database on the use of ECT in schizophrenia, when used as a monotherapy for schizophrenia, ECT is more effective than sham ECT, and this mostly stems from improvement in the positive symptoms. This supports the historical and economical (poor access to medications) use of ECT for schizophrenia as mentioned above. However, when compared with medications, ECT is inferior in outcome to antipsychotic medications [12].

Tharyan and Adams' conclusions were supported by two successive meta-

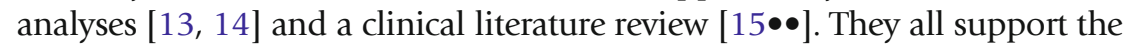
hypothesis that ECT's effect on schizophrenia outcome may be specific to the positive symptoms of schizophrenia and is likely not due to decreasing affective symptoms in patients with questionable diagnosis of schizophrenia (e.g., bipolar disorder with psychotic features). According to Zervas et al. [15••], ECT is particularly effective in improving auditory hallucinations, delusions, and thought insertion. Unfortunately, the mechanism by which ECT produces this therapeutic effect is unknown.

The effect of ECT on negative symptoms is more challenging to analyze than the positive symptoms using subscales or negative symptoms scales because of the following: (a) overlap with depressive mood symptoms, (b) the use of antipsychotic medication in combination treatment trials, and (c) the duration of illness and the difficulty to control for this as a confounding factor in trials [12, $15 \bullet \bullet, 16,17]$.

There are no medication-free, randomized, prospective ECT trials studying its effects on negative symptoms. Nevertheless, some adjunctive trials where this 
was analyzed or reported showed either no change or worsening of negative symptoms $[12,15 \bullet \bullet]$ such as one study from Thailand that investigated the combination of ECT and flupenthixol [18]. Other open-label, non-randomized studies have reported small but statistically significant subscale improvement in negative symptoms [19-21]. The clinical significance of this improvement,

however, remains unconvincing.

ECT has been most consistently shown to be an effective adjunct treatment for schizophrenia patients who are partially responsive or refractory to Clozapine treatment. Clozapine can induce seizures at higher doses, but it is unknown if this effect synergizes with the augmentation effect obtained from ECT.

Surprisingly few clinical trials were performed investigating the efficacy of using ECT in combination with antipsychotic medications. Moreover, many of them were open-label trials, and the results compared to those of the blinded trials disagreed dramatically: the open label trials were overwhelmingly positive in contrast to the blinded trials which were inconclusive. However, there were methodological issues (e.g., statistical power and treatment parameters such as the duration of ECT) with the blinded studies; therefore, clinicians did not abandon this approach. These earlier studies were reviewed by Braga and Petrides [22] for antipsychotic medications and ECT and by Havaki-Kontaxaki et al. [23] for Clozapine and ECT.

More recently, Masoudzadeh and Khalilian matched 16 patients with schizophrenia to three arms: Clozapine, ECT, and Clozapine plus ECT. They demonstrated a $71 \%$ decrease in the combination arm, $46 \%$ decrease in the Clozapine arm, and $40 \%$ decrease in the ECT arm of Positive and Negative Syndrome Scale scores [24].

Encouraged by this report and by their own positive pilot trial, Petrides and colleagues launched the first prospective randomized controlled trial with masked raters to investigate the efficacy and tolerability of Clozapine augmen-

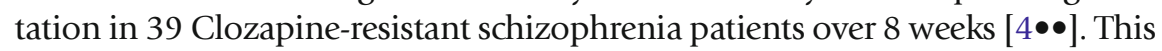
study was more rigorous than others: (i) treatment response-BPRS reduction-threshold was doubled to balance the labor intensiveness of ECT for patients, (ii) overlap with mood symptoms was carefully minimized by excluding schizoaffective disorder patients and patients with prominent depression symptoms, and (iii) patients received a robust ECT course (20 treatments over 8 weeks). None of the Clozapine patients achieved treatment response reflecting the efficacy of participant selection and they were crossed over to ECT and achieved $47 \%$ response. However, $50 \%$ of the Clozapine plus ECT patients showed response ( $60 \%$ by mainstream criterion), and strikingly, there were no group differences in neurocognitive measures or other adverse effects. The limitations of the study were the lack of sham ECT (unethical), lack of long-term follow-up, and low participant number although this was one of the largest studies in this field.

\section{Patient selection and ECT technique}

Although robust data are lacking, some investigators have proposed predictive factors for response to ECT in schizophrenia patients [25]. However, these criteria are similar to the factors for overall prognosis of schizophrenia and for overall 
response to antipsychotic medications (e.g., affective symptoms, negative family history, shorter duration of illness). Hence, they are unlikely to be specifically useful for the consideration of adjunct ECT for patients in clinical practice.

Similarly, there is little to guide specific treatment parameters for ECT in schizophrenia. Investigators transferred parameters used in depression treatment studies to schizophrenia treatment studies. Current consensus in clinical practice is to favor bilateral ECT treatments especially in more severe cases and in catatonias although successful uses of unilateral ECT have been reported.

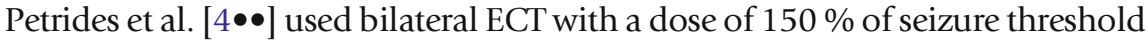
and bilateral ECT with doses of up to $400 \%$ of seizure threshold has been utilized by others.

In summary, ECT should be considered more often in patients with inadequate response to antipsychotic medications even when they do not have prominent affective symptoms. It should be initiated promptly when patients present with catatonia. ECT is unlikely to be beneficial for the negative symptoms, and its use for maintenance treatment is promising yet inadequately studied. It is well tolerated, and modern methods have improved tolerability and safety significantly. Its response predictors, optimal parameters, and action mechanism remain to be elucidated with further research.

\section{Transcranial magnetic stimulation}

Based on Faraday's principle of electromagnetic induction, TMS modulates the brain's electrical activity using very strong and rapidly alternating magnetic fields that pass through the scalp and the skull unimpeded. The magnetic field strength produced by TMS is 1.5-3 Teslas and is comparable to a diagnostic MRI, but the magnetic pulses target a focused area of the cortex and pulsate very rapidly. TMS can be administered in single pulses or as a brief series of pulses for research, diagnostic, and therapeutic purposes. In most therapeutic applications, several hundred to several thousand pulses are usually applied over a period of minutes to hours. These pulses can be delivered in a rapid-fire (i.e., 5$20 \mathrm{~Hz}$ ) fashion, enhancing cortical activity or intermittently (i.e., less than or equal to $1 \mathrm{~Hz}$ ), inhibiting cortical activity. This is called repetitive transcranial magnetic stimulation or "rTMS". In this review, we will use the term TMS. TMS is a very safe and well-tolerated procedure. The most common adverse effects are pain at the site of stimulation, muscle contraction during treatments, and headaches after the treatments. There is a risk of unintended seizures during treatments, but this is very low (less than $1 \%$ for non-epileptic patients receiving high frequency TMS) [26].

TMS, unlike ECT, has first been used therapeutically in depression, and three TMS devices have already received FDA clearance for the treatment of medication-resistant major depressive disorder. For schizophrenia, it has shown promise in the treatment of negative symptoms, medication-resistant auditory hallucinations, and working memory deficits. 
with schizophrenia) [27-31] and one of which (associated with schizophrenia) reported failure [32]. Therefore, considering the strength of the evidence base and the risks and complications associated with catatonia, ECT should remain the therapeutic neuromodulation of choice in catatonia especially when associated with schizophrenia.

Functional neuroimaging studies suggest hyperactivity of temporoparietal cortex (TPC) in the perisylvian areas in schizophrenia patients experiencing auditory hallucinations [33•]. Up to a fourth of schizophrenia patients' auditory hallucinations (AH) do not respond to antipsychotic medications, and alternative treatments such as psychotherapy are inadequate [34]. Therefore, applying low-frequency TMS to decrease the hyperactivity of the TPC (which is involved in the perception of speech) offered an exciting research opportunity. Starting in 1999 [35], this has led Hoffman and colleagues to do a succession of trials, the most recent of which [36•] randomized 83 patients who were stimulated with $1-\mathrm{Hz}$ pulses at a motor threshold level of $90 \%$ over fifteen sessions and 960 pulses. This was done alternatively on both hemispheres, and there was a booster session for the better-responding hemisphere in the last 5 days of the study. In spite of earlier positive results, in this trial, the severity of AH did not decrease compared to sham treatment, but AH frequency decreased and the Clinical Global Impression (CGI) scores increased significantly.

There have been 27 randomized controlled trials investigating the effect of TMS on the severity of AH or psychosis in schizophrenia patients. Twenty-five of these trials (several of which reported no benefit) were reviewed and meta-

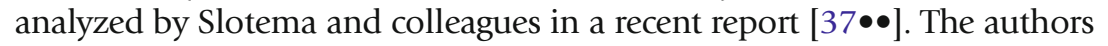
found an overall average effect size of 0.63 when the analysis was restricted to protocols utilizing $1 \mathrm{~Hz}$ TMS over the TPC. When other protocols were included, the effect size decreased considerably but remained modest implying that low-frequency TMS over TPC protocol is the most efficacious. Unlike with ECT, there was no significant improvement of the severity of overall psychosis with TMS treatments.

The two most recent randomized controlled trials were not included in this meta-analysis. The first one was inadequately powered and utilized a sham that was possibly active [38]. Interestingly, the second trial [39] was an extension of an earlier trial [40] whose statistical significance dissipated with the additional parameters and subjects. Neither of them demonstrated a significant difference between sham and active TMS protocols.

Overall, based on current evidence, there seems to be an adjunctive role for left-sided low-frequency TMS over the TPC to target treatmentresistant $\mathrm{AH}$ in schizophrenia. However, better-powered trials, further refinement, and optimization, and ideally with individualized parameters for patients, need to be achieved before accepting it as a therapeutic option into routine clinical care. 
symptoms lead to severe functional impairment and are the major cause of long-term disability in schizophrenia. Consequently, especially since ECT does not improve negative symptoms, TMS has been studied to target this symptom domain as an alternate plausible option.

The prevailing explanatory mechanism of negative symptoms is prefrontal cortex (PFC) hypoactivity, particularly involving the left side [41]. This has led to the proposal that this hypoactivity could be normalized by stimulating the dorsolateral prefrontal cortex (DLPFC) with high frequency TMS [42].

Cohen and colleagues performed the first trials of TMS for negative symptoms in six patients who were on antipsychotic medications for 3 months. After ten daily treatments over the PFC, they observed significant reduction in the Positive and Negative Syndrome Scale (PANSS) negative subscale scores. This was followed by several confirmatory open label trials by other investigators. The majority of randomized, shamcontrolled studies used high-frequency TMS over the left DLPFC. Four of these studies did not support a benefit from TMS for negative symptoms as assessed by the Scale for the Assessment of Negative Symptoms (SANS) and/or negative subscale of PANSS [43-46]. The largest and most recent trial involved 117 patients with prominent negative symptoms randomized to active TMS $(n=78)$ over left DLPFC or sham TMS $(n=39)$ and reported that active TMS improved PANSS negative subscale and SANS scores but did not affect CGI-S scores. The benefits persisted up to 24 weeks $[47 \bullet]$. A smaller $(n=32)$ recent study utilizing bilateral TMS also found improvement in SANS scores that lasted up to 3 months [48]. To date, four meta-analyses have been performed regarding the effect of TMS on negative symptoms and have consistently found a moderate effect size. This is most likely due to small number of participants in most of the studies. The most recent meta-analysis was reported in 2014 and calculated an effect size of 0.41 for PANSS and 0.80 for SANS out of 16 studies involving 342 participants [49•].

Similar to TMS for AH, TMS for negative symptoms offers hope and promise based on the meta-analyses and the low likelihood of publication bias. Larger studies with improved sham coil strategies will be needed to further investigate this modality. Using TMS for negative symptoms has a more plausible and parsimonious neurobiological mechanism underpinning its effect. Hence, we believe that it has a higher potential to be developed into a routinely used treatment modality for schizophrenia patients.

\section{Conclusion}

- ECT will continue to serve acutely and severely ill patients with schizophrenia. Its safety and tolerability can be further improved with continued research.

- TMS is in its earlier stages of development and shows great promise for further refinement and optimization. 
- TMS' tolerability and low risk profile facilitates clinical decisions of use in conjunction with ECT, medications and psychotherapy.

\section{Compliance with Ethics Guidelines}

\section{Conflict of Interest}

Mehmet E. Dokucu declares no conflict of interest.

Human and Animal Rights and Informed Consent

This article does not contain any studies with human or animal subjects performed by any of the authors.

\section{References and Recommended Reading}

Papers of particular interest, published recently, have been

highlighted as:

- Of importance

- Of major importance

1. Brenner HD, Dencker SJ, Goldstein MJ, Hubbard JW, Keegan DL, Kruger G, et al. Defining treatment refractoriness in schizophrenia. Schizophr Bull. 1990;16(4):551-61.

2. Lieberman JA, Stroup TS, McEvoy JP, Swartz MS, Rosenheck RA, Perkins DO, et al. Effectiveness of antipsychotic drugs in patients with chronic schizophrenia. N Engl J Med. 2005;353(12):1209-23. doi:10.1056/ NEJMoa051688.

3. Lieberman JA, Safferman AZ, Pollack S, Szymanski S, Johns C, Howard A, et al. Clinical effects of clozapine in chronic schizophrenia: response to treatment and predictors of outcome. Am J Psychiatry. 1994;151(12):1744-52.

4.• Petrides G, Malur C, Braga RJ, Bailine SH, Schooler NR, Malhotra AK, et al. Electroconvulsive therapy augmentation in clozapine-resistant schizophrenia: a prospective, randomized study. Am J Psychiatry. 2015;172(1):52-8. doi:10.1176/appi.ajp.2014.13060787.

A large and rigorously conducted study with impressive results.

5. Leiknes KA, Jarosh-von Schweder L, Hoie B. Contemporary use and practice of electroconvulsive therapy worldwide. Brain and Behav. 2012;2(3):283-344. doi:10.1002/brb3.37.

6. Chanpattana W, Andrade C. ECT for treatmentresistant schizophrenia: a response from the far East to the UK NICE report. J ECT. 2006;22:4-12.

7. Fink M, Taylor MA. The catatonia syndrome: forgotten but not gone. Arch Gen Psychiatry. 2009;66(11):11737. doi:10.1001/archgenpsychiatry.2009.141.

8. Daniels J. Catatonia: clinical aspects and neurobiological correlates. J Neuropsychiatry Clin Neurosci. 2009;21(4):371-80. doi:10.1176/appi.neuropsych.21. 4.371 .

9. Escobar R, Rios A, Montoya ID, Lopera F, Ramos D, Carvajal C, et al. Clinical and cerebral blood flow changes in catatonic patients treated with ECT. J Psychosom Res. 2000;49(6):423-9.

10. Rohland BM, Carroll BT, Jacoby RG. ECT in the treatment of the catatonic syndrome. J Affect Disord. 1993;29(4):255-61.

11. Suzuki K, Awata S, Takano T, et al. Improvement of psychiatric symptoms after electroconvulsive therapy in young adults with intractable first-episode schizophrenia and schizophreniform disorder. J Exp Med. 2006;210:213-20.

12. Tharyan $P$, Adams CE. Electroconvulsive therapy for schizophrenia. Cochrane Database Syst Rev. 2005;2, CD000076. doi:10.1002/14651858.CD000076.pub2.

13. Painuly N, Chakrabarti S. Combined use of electroconvulsive therapy and antipsychotics in schizophrenia: the Indian evidence. A review and a meta-analysis. J ECT. 2006;22:59-66.

14. Matheson SL, Green MJ, Loo C, Carr VJ. Quality assessment and comparison of evidence for electroconvulsive therapy and repetitive transcranial magnetic stimulation for schizophrenia: a systematic meta-review. Schizophr Res. 2010;118:201-10.

$15 . \bullet$ Zervas IM, Theleritis C, Soldatos CR. Using ECT in schizophrenia: a review from a clinical perspective. World J Biol Psy Off J World Fed Soc Biol Psyc. 2012;13(2):96105. doi:10.3109/15622975.2011.564653.

A comprehensive review for clinicians considering ECT.

16. Meltzer HY. Dimensions of outcome with clozapine. $\mathrm{Br}$ J Psychiatry Suppl. 1992;17:46-53.

17. Kane JM. Tools to assess negative symptoms in schizophrenia. J Clin Psychiatry. 2013;74(6), e12. doi:10.4088/JCP.12045tx2c. 
18. Chanpattana W, Somchai Chakrabhand ML. Combined ECT and neuroleptic therapy in treatment- refractory schizophrenia: prediction of outcome. Psychiatry Res. 2001;105:107-15.

19. Meltzer HY. Treatment of the neurolepticnonresponsive schizophrenic patient. Schizophr Bull. 1992;18(3):515-42.

20. Garg R, Chavan BS, Arun P. Quality of life after electroconvulsive therapy in persons with treatment resistant schizophrenia. Indian J Med Res. 2011;133:641-4.

21. Tang WK, Ungvari GS. Efficacy of electroconvulsive therapy combined with antipsychotic medication in treatment-resistant schizophrenia: a prospective, open trial. J ECT. 2002;18(2):90-4.

22. Braga RJ, Petrides G. The combined use of electroconvulsive therapy and antipsychotics in patients with schizophrenia. J ECT. 2005;21(2):75-83.

23. Havaki-Kontaxaki BJ, Ferentinos PP, Kontaxakis VP, Paplos KG, Soldatos CR. Concurrent administration of clozapine and electroconvulsive therapy in clozapineresistant schizophrenia. Clin Neuropharmacol. 2006;29(1):52-6.

24. Masoudzadeh A, Khalilian AR. Comparative study of clozapine, electroshock and the combination of ECT with clozapine in treatment-resistant schizophrenic patients. Pak J Biol Sci PJBS. 2007;10(23):4287-90.

25. Chanpattana W, Sackeim HA. Electroconvulsive therapy in treatment-resistant schizophrenia. J ECT. 2010;26(4):289-98.

26. Rossi S, Hallett M, Rossini PM, Pascual-Leone A. Safety of TMSCG. Safety, ethical considerations, and application guidelines for the use of transcranial magnetic stimulation in clinical practice and research. Clin Neurophysiol. 2009;120(12):2008-39.

27. Koppi S, Conca A, Swoboda E, Konig P. Transcranial magnetic stimulation in depressed patients: a new antidepressive therapeutic principle? Presentation of a pilot trial. Wien Med Wochenschr. 1996;146(3):49-54.

28. Grisaru N, Chudakov B, Yaroslavsky Y, Belmaker RH. Catatonia treated with transcranial magnetic stimulation. Am J Psychiatry. 1998;155(11):1630.

29. Saba G, Rocamora JF, Kalalou K, Benadhira R, Plaze M, Aubriot-Delmas B, et al. Catatonia and transcranial magnetic stimulation. Am J Psychiatry. 2002;159(10):1794.

30. Di Michele V, Bolino F. A novel treatment option of bipolar depression with psychotic and catatonic features. Gen Hosp Psychiatry. 2006;28(4):364-5. doi:10. 1016/j.genhosppsych.2006.05.003.

31. Kate MP, Raju D, Vishwanathan V, Khan FR, Nair, Thomas SV. Successful treatment of refractory organic catatonic disorder with repetitive transcranial magnetic stimulation (rTMS) therapy. J Neuropsychiatry Clin Neurosci. 2011;23(3):E2-3. doi:10.1176/appi. neuropsych.23.3.E2.

32. Trojak B, Meille V, Bonin B, Chauvet-Geliner JC. Repetitive transcranial magnetic stimulation for the treatment of catatonia: an alternative treatment to electroconvulsive therapy? J Neuropsychiatry Clin Neurosci. 2014;26(2):E42-3. doi:10.1176/appi. neuropsych.13050102.

33. Garcia-Marti G, Aguilar EJ, Marti-Bonmati L, Escarti MJ, Sanjuan J. Multimodal morphometry and functional magnetic resonance imaging in schizophrenia and auditory hallucinations. World J Radiol. 2012;4(4):15966. doi:10.4329/wjr.v4.i4.159.

A concise review of the imaging markers for auditory hallucinations.

34. Shergill SS, Murray RM, McGuire PK. Auditory hallucinations: a review of psychological treatments. Schizophr Res. 1998;32(3):137-50.

35. Hoffman RE, Boutros NN, Berman RM, et al. Transcranial magnetic stimulation of left temporoparietal cortex in three patients reporting hallucinated "voices". Biol Psychiatry. 1999;46:130-2.

36. Hoffman RE, Wu K, Pittman B, et al. Transcranial magnetic stimulation of Wernicke's and right homologous sites to curtail "voices": a randomized trial. Biol Psychiatry. 2013;73:1008-14.

More recent, better-powered study with modest results.

37.• Slotema CW, Blom JD, van Lutterveld R, Hoek HW, Sommer IE. Review of the efficacy of transcranial magnetic stimulation for auditory verbal hallucinations. Biol Psychiatry. 2014;76(2):101-10. doi:10.1016/j. biopsych.2013.09.038.

A comprehensive review of TMS for positive symptoms.

38. Kim EJ, Yeo S, Hwang I, Park JI, Cui Y, Jin HM, et al. Bilateral repetitive transcranial magnetic stimulation for auditory hallucinations in patients with schizophrenia: a randomized controlled, cross-over study. Clin Psychopharm Neurosci. 2014;12(3):222-8. doi:10.9758/cpn. 2014.12.3.222.

39. Bais L, Vercammen A, Stewart R, van Es F, Visser B, Aleman A, et al. Short and long term effects of left and bilateral repetitive transcranial magnetic stimulation in schizophrenia patients with auditory verbal hallucinations: a randomized controlled trial. PLoS One. 2014;9(10), e108828. doi:10.1371/journal.pone. 0108828 .

40. Vercammen A, Knegtering H, Bruggeman R, Westenbroek HM, Jenner JA, Slooff CJ, et al. Effects of bilateral repetitive transcranial magnetic stimulation on treatment resistant auditory-verbal hallucinations in schizophrenia: a randomized controlled trial. Schizophr Res. 2009;114(1-3):172-9. doi:10.1016/j. schres.2009.07.013.

41. Siever LJ, Davis KL. The pathophysiology of schizophrenia disorders: perspectives from the spectrum. Am J Psychiatry. 2004;161(3):398-413.

42. Paus T. Imaging the brain before, during, and after transcranial magnetic stimulation. Neuropsychologia. 1999;37(2):219-24.

43. Holi MM, Eronen M, Toivonev K, Toivonen P, Marttunen M, Naukkarinen H. Left prefrontal repetitive transcranial magnetic stimulation in schizophrenia. Schizophr Bull. 2004;30(2):429-34. 
44. Novak T, Horacek J, Mohr P, et al. The double-blind sham-controlled study of high-frequency rTMS $(20 \mathrm{~Hz})$ for negative symptoms in schizophrenia: negative results. Neuro Endocrinol Lett. 2006;27(1-2):209-13.

45. Fitzgerald PB, Herring S, Hoy K, McQueen S, Segrave R, Kulkarni J, et al. A study of the effectiveness of bilateral transcranial magnetic stimulation in the treatment of the negative symptoms of schizophrenia. Brain Stimul. 2008;1(1):27-32. doi:10.1016/j.brs.2007.08.001.

46. Barr MS, Farzan F, Tran LC, Fitzgerald PB, Daskalakis ZJ. A randomized controlled trial of sequentially bilateral prefrontal cortex repetitive transcranial magnetic stimulation in the treatment of negative symptoms in schizophrenia. Brain Stimul. 2012;5(3):337-46. doi:10.1016/j.brs.2011.06.003.

47. Quan WX, Zhu XL, Qiao H, Zhang WF, Tan SP, Zhou $\mathrm{DF}$, et al. The effects of high-frequency repetitive transcranial magnetic stimulation (rTMS) on negative symptoms of schizophrenia and the follow-up study. Neurosci Lett. 2015;584:197-201. doi:10.1016/j. neulet.2014.10.029.

The most recent and largest study to date of TMS use for negative symptoms.

48. Dlabac-de Lange JJ, Bais L, van Es FD, Visser BG, Reinink E, Bakker B, et al. Efficacy of bilateral repetitive transcranial magnetic stimulation for negative symptoms of schizophrenia: results of a multicenter doubleblind randomized controlled trial. Psychol Med. 2015;45(6):1263-75. doi:10.1017/ S0033291714002360.

49.• Shi C, Yu X, Cheung EF, Shum DH, Chan RC. Revisiting the therapeutic effect of rTMS on negative symptoms in schizophrenia: a meta-analysis. Psychiatry Res. 2014;215(3):505-13. doi:10.1016/j.psychres.2013.12. 019.

Comprehensive, up-to-date review and metanalysis. 